\title{
Análise das atividades farmacológicas da Handroanthus serratifolius (Vahl) S.Grose
}

\author{
Analysis of the pharmacological activities of Handroanthus serratifolius (Vahl) S.Grose \\ Análisis de las actividades farmacológicas de Handroanthus serratifolius (Vahl) S.Grose
}

Recebido: 05/11/2021 | revisado: 12/11/2021 | aceito: 16/01/2022 | publicado: 18/01/2022

Fernanda Arias de Almeida Macêdo

ORCID: https://orcid.org/0000-0002-7948-8015

Centro Universitário UNIFACID, Brasil

E-mail: ariasfernandinha@gmail.com

Mayara Ladeira Coêlho

ORCID: https://orcid.org/0000-0002-8084-5964 Centro Universitário UNIFACID, Brasil

E-mail: mayara.coelho@professores.facid.edu.br

Manoela Cavalcante Ribeiro

ORCID: https://orcid.org/0000-0003-0785-4810 Centro Universitário UNIFACID, Brasil

E-mail: manoelacavalcanteribeiro@hotmail.com

Elcio Daniel Sousa Barros

ORCID: https://orcid.org/0000-0001-9837-2248

Instituto Federal do Maranhão, Brasil

E-mail: elcio.barros@ifma.edu.br

João Paulo da Silva Gomes

ORCID: https://orcid.org/0000-0003-0160-5514 Universidade Federal do Piauí, Brasil

E-mail: pjoao523@gmail.com

Ceres Lima Batista

ORCID: https://orcid.org/0000-0001-6465-2292 Universidade Federal do Piaú, Brasil

E-mail: cereslbat@gmail.com

Layana Karine Farias Lima

ORCID: https://orcid.org/0000-0002-1431-2422

Universidade Federal do Piauí, Brasil

E-mail: Layana_farias@hotmail.com

Renato Pinto de Sousa

ORCID: https://orcid.org/0000-0002-8217-3655

Universidade Federal do Piauí, Brasil

E-mail: renatopintto@hotmail.com

\begin{abstract}
Resumo
Handroanthus serratifolius (Vahl) S.Grose, (sin. Handroanthus albus; sin. Tabebuia serratifolia), conhecida popularmente como ipê-amarelo, pertence à família Bignoniaceae. Plantas dessa família, possuem diversas atividades farmacológicas descritas na literatura, as quais ocorrem devido à presença de metabólitos secundários como, por exemplo, o lapachol, que induz o estresse oxidativo em células e pode ser eficaz contra parasitas intracelulares obrigatórios e células de câncer. O objetivo do artigo foi analisar as propriedades farmacológicas presentes nos compostos químicos isolados de diferentes partes da $H$. serratifolius (Vahl) S.Grose, (sin. H. albus; sin. T. serratifolia). O estudo trata-se de uma revisão de literatura do tipo integrativa, realizada nas bases de dados Science Direct, Google Scholar, Embase, MEDLINE via PubMed, Web of Science e na Biblioteca Virtual de Saúde (BVS), a partir de trabalhos publicados nos últimos seis anos, nos idiomas português e inglês, que tenham abordado na íntegra o tema em pesquisa. Observou-se nos estudos analisados que as espécies de ipê amarelo possuem atividades antibacteriana, antinociceptiva, antifúngica, antinflámatória, antiparasitária, citotóxica, antileishmanicida, e que essas atividades podem ser encontradas em extratos de folhas e no caule. Além disso, o lapachol mostrou-se responsável por algumas dessas propriedades farmacológicas. Portanto, pode-se concluir que a Handroanthus serratifolius é uma fonte potencial para pesquisas e desenvolvimento de novas substâncias com diferentes aplicabilidades farmacológicas.
\end{abstract}

Palavras-chave: Handroanthus serratifolius (Vahl) S.Grose; Handroanthus albus; Tabebuia serratifolia; Atividade farmacológica; Lapachol. 


\begin{abstract}
Handroanthus serratifolius (Vahl) S.Grose, (syn. Handroanthus albus; syn. Tabebuia serratifolia), popularly known as yellow-ipê, belongs to the Bignoniaceae family. Plants of this family have several pharmacological activities described in the literature, which occur due to the presence of secondary metabolites, such as lapachol, which induces oxidative stress in cells and can be effective against obligate intracellular parasites and cancer cells. The aim of the article was to analyze the pharmacological properties present in chemical compounds isolated from different parts of H. serratifolius (Vahl) S.Grose, (syn. H. albus; syn. T. serratifolia). The study is an integrative literature review, carried out in the databases Science Direct, Google Scholar, Embase, MEDLINE via PubMed, Web of Science and the Virtual Health Library (VHL), based on works published in the last six years, in Portuguese and English, that have fully addressed the topic under investigation. It was observed in the analyzed studies that the species of yellow ipe have antibacterial, antinociceptive, antifungal, antiinflammatory, antiparasitic, cytotoxic, antileishmanial and antiplasmoid activities, and that these activities can be found in leaf and stem extracts. Furthermore, lapachol has been shown to be responsible for some of these pharmacological properties. Therefore, it can be concluded that Handroanthus serratifolius is a potential source for research and development of new substances with different pharmacological applicability.

Keywords: Handroanthus serratifolius (Vahl) S.Grose; Handroanthus albus; Tabebuia serratifolia; Pharmacological activity; Lapachol.
\end{abstract}

\title{
Resumen
}

Handroanthus serratifolius (Vahl) S. Grose, (sin. Handroanthus albus; sin. Tabebuia serratifolia), planta de la familia Bignoniaceae, conocida popularmente como yellow-ipê. Las plantas de la familia Bignoniaceae, tienen varias actividades farmacológicas descritas en la literatura. Estas propiedades se deben a la presencia de metabolitos secundarios, como el lapachol, que induce estrés oxidativo en las células y puede ser eficaz contra parásitos intracelulares obligados y células cancerosas. Así, las plantas emergen como fuentes importantes en la investigación y desarrollo de nuevas alternativas terapéuticas o en la mejora de tratamientos existentes. Así, el objetivo del artículo fue analizar las propiedades farmacológicas presentes en compuestos químicos aislados de diferentes partes de $H$. serratifolius (Vahl) S.Grose, (sin. $H$. albus; sin. T. serratifolia). El estudio es una revisión integradora de la literatura, realizada en las bases de datos Science Direct, Google Scholar, Embase, MEDLINE vía PubMed, Web of Science y la Virtual Health Library (VHL), a partir de trabajos publicados en los últimos seis años, en portugués e inglés, que han abordado en su totalidad el tema investigado. En los estudios analizados se observó que las especies de ipe amarillo tienen actividades antibacterianas, antinociceptivas, antifúngicas, antiinflamatorias, antiparasitarias, citotóxicas, antileishmaniales y antiplasmoides, y que estas actividades se pueden encontrar en extractos de hojas y tallos. Además, se ha demostrado que el lapachol es responsable de algunas de estas propiedades farmacológicas. Por tanto, se puede concluir que Handroanthus serratifolius es una fuente potencial de investigación y desarrollo de nuevas sustancias con diferente aplicabilidad farmacológica.

Palabras clave: Handroanthus serratifolius (Vahl) S.Grose; Handroanthus albus; Tabebuia serratifolia; Actividad farmacológica; Lapachol.

\section{Introdução}

A Handroanthus serratifolius (Vahl) S.Grose, (sin. Handroanthus albus; sin. Tabebuia serratifolia) é uma espécie florestal nativa de grande importância em função de suas utilidades econômicas. Pertence a família Bignoniaceae, popularmente conhecida como ipê-amarelo, ipê-tabaco, pau-d'arco-amarelo. É uma árvore que pode medir até 30 metros de altura, de ramos cilíndricos e folhas 5-7-folioladas (Reflora, 2015). A espécie possui grande valor comercial e é comumente utilizada como planta ornamental na arborização urbana, e por sua madeira ser considerada dura, devido a alta densidade, é considerada boa fonte para produção de móveis (Sibbr, 2021).

A família Bignoniaceae é reconhecida na literatura pela variedade de plantas que apresentam atividades farmacológicas bem estabelecidas, como é o caso da Oroxylum indicum (L.) que demonstrou atividade hepatoprotetora por reduzir o estresse oxidativo (Mohan et al., 2016), atenuou os sintomas de colite ulcerativa em ratos (Joshi et al., 2011), além de possuir metabólitos secundários com atividade antibacteriana e anticancerígena (Buranrat et al., 2020). A espécie Arribidaea chica mostrou potencial anti-inflamatório da osteoartrite, antinociceptivo e atividade contra o câncer de mama em animais (Vasconcelos et al., 2019; Rocha et al., 2011; Rocha et al., 2019). Espécies de Tecoma, em estudos in vitro, evidenciaram atividade contra o Zika vírus (Reis et al., 
2020). A Jacaranda decurrens Cham exibiu atividade cicatrizante de feridas cutâneas (Serra et al., 2020). Enquanto a Cecropia pachystachya apresentou atividade antibacteriana a partir de extratos aquosos e etanólicos contra cepas de Staphylococcus aureus (Costa \& Hoscheid, 2018).

Os compostos químicos comumente isolados das plantas da família Bignoneaceae, aos quais são atribuídas as mais variadas atividades farmacológicas, incluem os iridóides, ácidos fenólicos, fenilpropanoides, triterpenos, lignanos e neolignanos, isocumarinas, flavanóides, acetosídeos, quinonas e naftoquinonas (Zhang et al., 2016; Piaz et al., 2013; Sichaem et al., 2012; Moharram \& Marzouk, 2007). Na espécie da Handroanthus serratifolius destaca-se o lapachol, uma naftoquinona, que possui um perfil químico caracterizado pela capacidade de induzir o estresse oxidativo em células, a partir da formação de espécies reativas de oxigênio (ROS), levando a danos intracelulares de proteínas e do DNA, mostrando-se como uma substância promissora, especialmente, contra parasitas intracelulares obrigatórios ou na desregulação da homeostasia de células de câncer (Da Silva et al., 2003; Bolton et al., 2000; Fiorito et al., 2014).

Desta forma, os metabólitos secundários das plantas dessa família demonstram promissora fonte de novas alternativas terapêuticas, seja de forma intrínseca ou na modulação positiva do efeito de fármacos utilizados rotineiramente na clínica, a partir de mecanismos que interferem na expressão de mediadores do processo inflamatório, na produção de segundos mensageiros e perturbações na expressão de fatores de transcrição (Filardi et al., 2018; Joly et al, 2011; Calixto, 2005). Logo, esse estudo tem como objetivo analisar, a partir de uma revisão integrativa da literatura, as atividades farmacológicas descritas da Handroanthus serratifolius.

\section{Metodologia}

\section{Tipo de estudo}

O estudo trata-se de uma revisão de literatura do tipo integrativa utilizando uma abordagem qualitativa de estudos de avaliação da atividade farmacológica de Handroanthus serratifolius (Vahl) Grose, Tabebuia serratifolia (Vahl) Nicholson e Handroanthus albus, popularmente conhecidos como Ipê-amarelo.

\section{Estratégia de busca nas bases de dados}

A busca avançada foi realizada entre os meses de abril e maio de 2021, nas bases de dados Science Direct, Embase, MEDLINE via PubMed, Google Scholar, Web of Science e na Biblioteca Virtual de Saúde (BVS). Os descritores selecionados - de acordo com o DeCS/MeSH - foram: "Handroanthus serratifolius (Vahl) Grose", "Handroanthus serratifolius (Vahl)" "Tabebuia serratifolia (Vahl) Nicholson" "Handroanthus albus" "atividade farmacológica”, "pharmacology activity”, e foi utilizado o operador booleano “AND” na interseção dos descritores.

\section{Critérios de inclusão e exclusão}

Os critérios de inclusão adotados foram: artigos publicados nos últimos 6 anos, que avaliassem a atividade farmacológica da Handroanthus serratifolius (Vahl) Grose, Tabebuia serratifolia (Vahl) Nicholson e Handroanthus albus, em modelos de estudos in vitro ou in vivo. Foram considerados artigos em português e inglês.

Foram excluídos artigos que não abordassem de diretamente a temática, bem como trabalhos que não apresentavam textos na íntegra.

\section{Resultados e Discussão}


Research, Society and Development, v. 11, n. 2, e5611222891, 2022

(CC BY 4.0) | ISSN 2525-3409 | DOI: http://dx.doi.org/10.33448/rsd-v11i2.22891

Foram selecionados 8 artigos que atenderam aos critérios previamente definidas para serem analisados na íntegra. De um total de 15 artigos, 7 foram excluídos por não abordarem o temo em estudo e por terem sido publicados fora do período selecionado para a pesquisa. Os artigos selecionados que possuem resultados sobre atividade farmacológica da Handroanthus serratifolius (Vahl) S. Grose publicados entre 2015 e 2020, foram organizados no Quadro 1.

Os estudos selecionados analisaram a atividade antifúngica do extrato etanólico das folhas, do caule e da raiz (Almeida et al., 2015), atividade antileishmania do extrato etanólico do caule (Costa et al., 2017), atividade citotóxica, antioxidante ou antimicrobiana do extrato hidrometanólico das flores e atividade citotóxica de polissacarídeos extraídos da casca do caule (Carlotto et al., 2020), atividade antinociceptiva e antiinflamatória do extrato aquoso liofilizado das folhas (Maria-ferreira et al., 2020). 
Quadro 1: Estudos de avaliação da atividade farmacológica de Handroanthus serratifolius (Vahl) S. Grose. Tipos de extratos e partes da Handroanthus serratifolius (Vahl) S.Grose, (sin. Handroanthus albus; sin. Tabebuia serratifolia) utilizados em estudos de avaliação de suas atividades farmacológicas.

\begin{tabular}{|c|c|c|c|c|c|}
\hline AUTOR & ANO & TÍTULO & $\begin{array}{l}\text { ATIVIDADE } \\
\text { FARMACOLÓGICA }\end{array}$ & $\begin{array}{l}\text { TIPO DE EXTRATO/ SUBSTÂNCIA } \\
\text { ISOLADA }\end{array}$ & $\begin{array}{l}\text { PARTE } \\
\text { UTILIZADA }\end{array}$ \\
\hline $\begin{array}{l}\text { Almeida } \\
\text { et al. }\end{array}$ & 2015 & $\begin{array}{l}\text { Atividade Inibitória de Handroanthus serratifolius sobre } \\
\text { Candida albicans }\end{array}$ & Atividade antifúngica & Extrato etanólico & $\begin{array}{l}\text { Folhas, caule e } \\
\text { raiz }\end{array}$ \\
\hline $\begin{array}{l}\text { Costa et } \\
\text { al. }\end{array}$ & 2017 & $\begin{array}{l}\text { Antileishmanial activity of Handroanthus serratifolius } \\
\text { (Vahl) S. Grose (Bignoniaceae) }\end{array}$ & Atividade antileishmania & Extrato etanólico & Caule \\
\hline $\begin{array}{l}\text { Barcelos } \\
\text { et al. }\end{array}$ & 2017 & $\begin{array}{l}\text { Análise fitoquímica e das atividades citotóxica, } \\
\text { antioxidante, e antibacteriana das flores de Tabebuia } \\
\text { serratifolia (Vahl) Nicholson }\end{array}$ & $\begin{array}{l}\text { Atividades citotóxica, antioxidante } \\
\text { e antibacteriana }\end{array}$ & Extrato hidrometanólico & Flores \\
\hline $\begin{array}{l}\text { Brandão } \\
\text { et al. }\end{array}$ & 2018 & $\begin{array}{l}\text { Antimalarial naphthoquinones. Synthesis via click } \\
\text { chemistry, in vitro activity, docking to PfDHODH and SAR } \\
\text { of lapachol-based compounds Antimalarial } \\
\text { naphthoquinones. Synthesis via click chemistry, in vitro } \\
\text { activity, docking to PfDHODH and SAR of lapachol-based } \\
\text { compounds }\end{array}$ & Atividade antimalária & $\begin{array}{l}\text { O produto natural lapachol foi isolado por } \\
\text { processamento básico / ácido }\end{array}$ & Caule \\
\hline $\begin{array}{l}\text { Costa et } \\
\text { al. }\end{array}$ & 2018 & $\begin{array}{l}\text { Avaliação da atividade esquistossomicida do lapachol e } \\
\text { análogos }\end{array}$ & Atividade antiparasitária & $\begin{array}{l}\text { Lapachol foi isolado através de cromatografia } \\
\text { em coluna de sílica gel }\end{array}$ & $\begin{array}{l}\text { Cascas do } \\
\text { caule }\end{array}$ \\
\hline $\begin{array}{l}\text { Melo et } \\
\text { al. }\end{array}$ & 2019 & $\begin{array}{l}\text { Antifungal and Antiplasmodial Activity of Isolated } \\
\text { Compounnd from Handroanthus serratifolius (Vahl) S. } \\
\text { Grose Sawdusts }\end{array}$ & $\begin{array}{l}\text { Atividades antifúngica } \\
\text { antiplasmodial }\end{array}$ & Extrato metanólico & $\begin{array}{ll}\text { Resíduos } & \text { de } \\
\text { madeira } & \text { do } \\
\text { caule } & \end{array}$ \\
\hline $\begin{array}{l}\text { Maria- } \\
\text { Ferreira et } \\
\text { al }\end{array}$ & 2020 & $\begin{array}{l}\text { A polysaccharide fraction from Handroanthus albus } \\
\text { (yellow ipê) leaves with antinociceptive and anti- } \\
\text { inflammatoryactivities }\end{array}$ & $\begin{array}{l}\text { Atividades antinociceptiva } \mathrm{e} \\
\text { antiinflamatória }\end{array}$ & Extrato aquoso liofilizado & Folhas \\
\hline $\begin{array}{l}\text { Carlotto et } \\
\text { al }\end{array}$ & 2020 & $\begin{array}{l}\text { Polysaccharide fractions from Handroanthus heptaphyllus } \\
\text { and Handroanthus albus barks: Structural } \\
\text { characterization and cytotoxic activity }\end{array}$ & $\begin{array}{l}\text { Atividade citotóxica em células de } \\
\text { câncer de mama humano }\end{array}$ & $\begin{array}{l}\text { Polissacarídeos extraídos com água fervente e } \\
\text { fracionados por processo de congelamento- } \\
\text { descongelamento }\end{array}$ & $\begin{array}{l}\text { Cascas } \\
\text { caule }\end{array}$ \\
\hline
\end{tabular}

Fonte: Autores. 
Plantas costumam produzir uma variedade de compostos conhecidos como fitoquímicos, responsáveis por manter as atividades fisiológicas do vegetal, como pela proteção contra agentes invasores, como fungos, insetos, bactérias e animais. Estes compostos vêm sendo utilizados como forma de tratar e curar diversas doenças (Dixon, 2001). Os estudos selecionados atribuíram às espécies de ipê-amarelo definidas, diversas atividades farmacológicas como antifúngica, citotóxica, antiparasitária, antibacteriana, dentre outras, ilustradas na Figura 1.

Figura1: Atividades farmacológicas da Handroanthus serratifolius (Vahl) S.Grose, (sin. Handroanthus albus; sin. Tabebuia serratifolia).

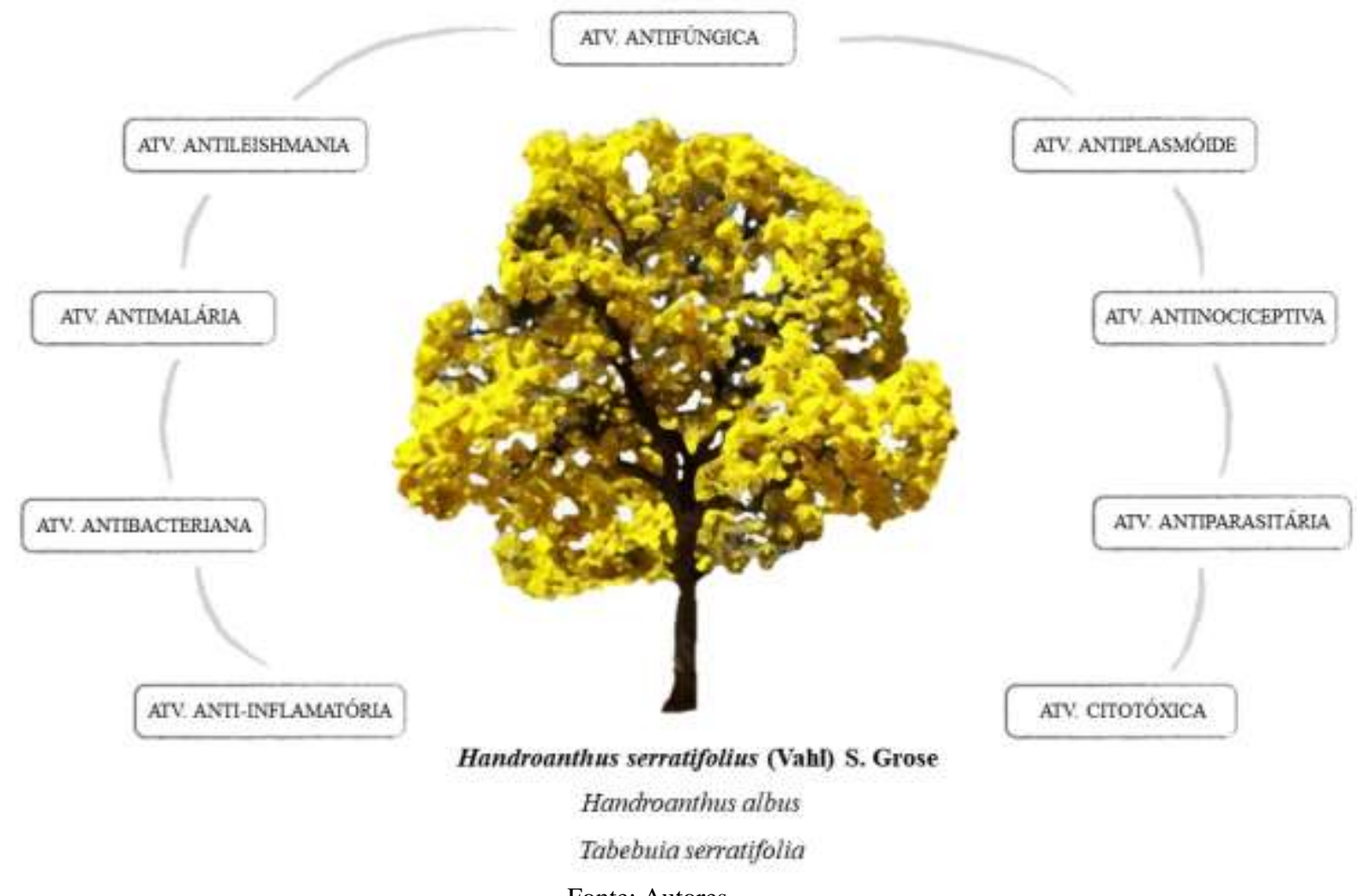

Fonte: Autores.

Dentre os artigos incluídos no estudo, a maioria utilizou-se do caule para a extração dos produtos farmacológicos. Boa parte dos processos extrativos foram realizados através de extrações alcoólicas e os potenciais farmacológicos foram avaliados in vitro.

Almeida et al. (2015) obteve extrato da planta Handroanthus serratifolius, através de extrato etanólico por meio de folhas, tronco e raiz conforme ilustrado na Tabela 2. Testes como determinação da concentração inibitória mínima (CIM) e sensibilidade em placas foram realizados de forma a atestar a eficácia da planta em inibir o crescimento do fungo Candida albicans. $\mathrm{O}$ autor observou redução no crescimento do fungo de maneira dose-dependente, demonstrando que o extrato possui potencial antifúngico contra a cepa utilizada. Outras espécies ligadas a família Bignoniaceae apresentaram potencial farmacológico antifúngico, como o encontrado por De Carvalho et al. (2007), através de estudo fitoquímico de Tynnanthus fasciculatus. Diversas espécies da família Bignoniaceae vem sendo testadas in vitro como potenciais antifúngicos, (Lucchese, 2006), corroborando com os resultados encontrados por Almeida et al. (2015).

Outra atividade farmacológica abordada nos estudos encontrados foi atividade citotóxica, estudada por Barcelos et al. (2017) e Carlotto et al. (2020). O primeiro autor utilizou-se de extrato hidrometanólico de flores da Tabebuia serratifolia, 
conforme ilustra a Tabela 2. Foram realizados análise fitoquímica, testes de citotoxicidade, de atividade antioxidante e de atividade antimicrobiana. Diversos fitoquímicos foram detectados, dentre eles flavonóides, saponinas, taninos, dentre outros. A citotoxicidade detectada no estudo (DL50 igual a $679 \mu \mathrm{g} / \mathrm{mL}$ ), apesar de evidenciar uma problemática em relação a segurança no consumo, pode indicar um potencial uso como antitumoral.

Ainda no estudo de alternativas quimioterápicas, Costa et al. (2018) avaliou potencial esquisstossomicida do lapachol e análogos, isolados do caule de Handroanthus serratifolius. Foram realizados experimentos in vitro frente a vermes adultos de Schistossoma mansoni, avaliando-se alterações na motilidade, morfologia e mortalidade através de microscopia óptica. O lapachol, in vitro, ao contrário do que mostraram outros estudos (Parrilha, 2012), não apresentou alterações nos vermes adultos. Tal fato pode ser explicado devido ao estágio em que o parasita se encontra. Alguns antiparasitários só são efetivos na destruição do parasita em um estágio específicos. Outra possibilidade seria o fato do lapachol poder ser considerado um prófarmaco, necessitando ser metabolizado para posterior transformação em substância antiparasitária, a $\beta$-lapachona, que, segundo o autor, levou a morte de 100\% dos parasitas, em 24 horas de tratamento em diferentes concentrações.

Costa et al. (2018) avaliou potencial do lapachol e análogos, isolados do caule de Handroanthus serratifolius. Foram realizados experimentos in vitro frente a vermes adultos de Schistossoma mansoni, avaliando-se alterações na motilidade, morfologia e mortalidade através de microscopia óptica. O lapachol, in vitro, ao contrário do que mostraram outros estudos (Parrilha, 2012), não apresentou alterações nos vermes adultos. Tal fato pode ser explicado devido ao estágio em que o parasita se encontra. Alguns antiparasitários só são efetivos na destruição do parasita em um estágio específicos. Outra possibilidade seria o fato do lapachol poder ser considerado um pró-farmaco, necessitando ser metabolizado para posterior transformação em substância antiparasitária, a $\beta$-lapachona, que, segundo o autor, levou a morte de $100 \%$ dos parasitas, em 24 horas de tratamento em diferentes concentrações

Carlotto et al. (2020) concluiu que, uma fração de extrato derivada de Handroanthus albus demonstrou potencial inibitório de crescimento de células tumorais malignas, evidenciando a necessidade de novos estudos in vitro do potencial citotóxico dessa planta, principalmente em relação a células cancerígenas. Resultados obtidos por Amâncio (2019) através da análise de extrato de espécies do gênero Jacaranda, também pertencentes a família Bignoniaceae, sugerem que algumas espécies podem ter potenciais fontes de moléculas citotóxicas, bem como possíveis usos como antitumoral.

Atividade antibacteriana vem sendo atribuída a diversas espécies do gênero Tabebuia. Extratos e frações de Tabebuia ochracea e Tabebuia rosea apresentaram resultados significativos na inibição de Staphylococcus aureus, responsável por infecções múltiplas e com resistência crescente a antibióticos. A atividade contra esses microorganismos pode ser relacionada a compostos de um natural quinóide, isolado e identificado em diversas espécies do gênero (Franco Ospina et al., 2013). Barcelos et al. (2017) verificou o potencial antimicrobiano de T. serratifolia contra microorganismos gram-positivos e gramnegativos, relacionando seus resultados a presença de flavonóides e triterpenos. O potencial de atividade antimicrobiana foi proporcional ao aumento das concentrações evidenciando as espécies de Tabebuia como alternativas terapêuticas para prevenção e tratamento de infecções bacterianas.

Brandão et al. (2018) avaliou um potencial antimalárico de derivados do lapachol, através de melhorias por modificações e sínteses químicas. Os compostos sintéticos apresentaram potencial superior ao lapachol, apresentando melhorias, principalmente em testes in vitro. Melo et al. (2019) estudou um potencial atividade antiplasmódica de três naftoquinonas isoladas de extrato de Handroanthus serratifolius, que apresentaram uma boa atividade contra Plasmodium falciparum.

Maria-Ferreira et al. (2020) realizou estudo avaliativo in vivo, utilizando-se de extrato de Handroanthus albus, isolando uma fração de polissacarídeo solúvel em água, posteriormente analisado quanto a características antinflamatórias. Como resultado, os autores concluíram que essa fração das folhas da planta apresentaram potencial antinociceptivo e atividade 
anti-inflamatória. A utilização desse gênero para fins medicinais vem sendo relatada desde os primórdios da humanidade sob forma de infusões, gargarejos, extratos e pós (Morais et al., 2020).

\section{Conclusão}

A partir dos estudos analisados, as espécies de ipês amarelo (Handroanthus serratifolius) demostraram propriedades farmacológicas contra cepas de Candida albicans, Staphylococcus aureus, inibição do crescimento de células cancerígenas de câncer de mama Apresentou também boa atividade contra Plasmodium falciparum, além de potenciais anti-inflamatório e antinociceptivo. As ações farmacológicas ao ipê amarelo são atribuídas a metabólitos secundários, que estão majoritariamente nas folhas e cascas do caule. Dentre estes, o lapachol mostrou-se como o composto responsável pela maioria das atividades farmacológicas reconhecidas para esta espécie. A Handroanthus serratifolius tem sido alvo de pesquisas nos últimos anos sendo, portanto, uma fonte natural potencial desenvolvimento de novas alternativas terapêuticas. Portanto seria interessante artigos com testes de citotoxidade em várias células diferentes.

\section{Referências}

Almeida, K. L., Silva, L. P., Silva, G. A., Neto, C. D. M. S., \& Prado, R. S. (2015). Atividade inibitória de Handroanthus serratifolius (Bignoniaceae) sobre Candida albicans. Revista Eletrônica da Faculdade de Ceres, 4(2).

Amâncio, E. A. M. (2019). Estudo fitoquímico de extratos etanólicos de espécies do gênero Jacaranda (Bignoniaceae) ocorrentes no Estado de Minas Gerais e avaliação da atividade citotóxica.

Barcelos, I. B., Bulian, A. L., Calazans, R. R. D. S. P., Degen, N. A., Alves, L. D. O., Sobral, F. D. O. S., \& Salvi, J. D. O. (2017). Análise fitoquímica e das atividades citotóxica, antioxidante, e antibacteriana das flores de Tabebuia serratifolia (Vahl) Nicholson. Revista Fitos, 11(1), 1-118.

Brandão, G. C., Missias, F. C. R., Arantes, L. M., Soares, L. F., Roy, K. K., Doerksen, R. J., \& Pereira, G. R. (2018). Antimalarial naphthoquinones. Synthesis via click chemistry, in vitro activity, docking to PfDHODH and SAR of lapachol-based compounds. European journal of medicinal chemistry, $145,191-205$.

Buranrat, B., Noiwetch, S., Suksar, T., \& Ta-ut, A. (2020). Inibition of cell proliferation and migration by Oroxylum indicum extracts on breast cancer cells via Rac1 modulation. J Pharm Anal, 10(2): 187-193.

Carlotto, J., de Almeida Veiga, A., de Souza, L. M, \& Cipriani, T. R. (2020). Polysaccharide fractions from Handroanthus heptaphyllus and Handroanthus albus barks: Structural characterization and cytotox.ic activity. International Journal of Biological Macromolecules, 165, 849-856.

Costa, E. V. S. (2018). Avaliação da atividade esquistossomicida do lapachol e análogos.

Costa, E. V. S., Brígido, H. P. C., Coelho-Ferreira, M. R., Brandão, G. C., \& Dolabela, M. F. (2017). Antileishmanial Activity of Handroanthus serratifolius (Vahl) S. Grose (Bignoniaceae). Evidence-Based Complementary and Alternative Medicine, 2017.

Calixto, J. B. (2005). Twenty-five years of research on medicinal plants in Latin America: A personal view. J. Ethnopharmacol. 100:131-134. 10.1016/j.jep.2005.06.004.

Da Costa, J. C. F., \& Hoscheid, J. (2018). Perfil fitoquímico e avaliação da atividade antimicrobiana de extratos aquoso e etanólico de folhas de Cecropia pachystachya. Revista Fitos, 12(2), 175-185.

Da Silva, M. N., \& Ferreira, V. F. M.C.B.V. (2003). Um panorama atual da química e da farmacologia de naftoquinonas, com ênfase na $\beta$-lapachona e derivados. Química Nova, 26(3), 407-416.

de Carvalho, C. A., de Oliveira, T. G., Vasconcelos, R., Manfré, R., de Freitas Andrade, D. C., da Matta, S. L. P., \& da Rosa, M. B. (2007). Estudo fitoquímico de Tynnanthus fasciculatus-Bignoniaceae. $47^{\circ}$ Congresso Brasileiro de Olericultura, Porto Seguro. Anais do Congresso Brasileiro de Olericultura, $25:$ 1-176.

de Melo, L. E. S., Cruz, K. S., Lima, P. I., Soares, C. C. D. N., de Souza, J. V. B., de Melo Marcelino, B. M., \& da Paz Lima, M (2019). Antifungal and Antiplasmodial Activity of Isolated Compounds from Handroanthus serratifolius (Vahl) S. Grose Sawdusts. Int. J. Adv. Res. Sci. Eng. Technol., 6.

Dixon, RA, (2001). Natural products and plant disease resistance. Nature, 411(6839), 843-847.

Franco Ospina, L. A., Castro Guerrero, J. P., Ocampo Buendía, Y. C., Pájaro Bolívar, I. B., \& Díaz Castillo, F. (2013). Actividad antiinflamatoria, antioxidante y antibacteriana de dos especies del género Tabebuia. Revista Cubana de Plantas Medicinales, 18(1), 34-46.

Filardi, F. L. R., de Barros F., Baumgratz J. F. A., Bicudo, C. E. M., Cavalcanti, T. B., Coelho, M. A. N., Costa, A. F., Costa, D. P., Goldenberg, R., Labiak, P. H., et al. Brazilian Flora 2020: Innovation and collaboration to meet Target 1 of the Global Strategy for Plant Conservation (GSPC) Rodriguésia. 69:15131527 .

Fiorito, S., Epifano, F., Bruyère, C., Mathieu, V., Kiss, R. S. (2014). Genovese Growth inhibitory activity for cancer cell lines of lapachol and its natural and semi-synthetic derivatives Bioorg. Med. Chem. Lett., 24, 454-457. 
Joly, C., Haddad, C., Verdade, L., Oliveira, M., Bolzani, V., \& Berlinck, R. (2011). Diagnóstico da pesquisa em biodiversidade no Brasil. Rev. USP. 89:114133. 10.11606/issn.2316-9036.v0i89p114-133.

Joshi, S. V., Vyas, B. A., Shah, P. D., Shah, D. R., Shah, S. A., \& Gandhi, T. R. (2011). Protective effect of aqueous extract of Oroxylum indicum Linn (root bark) against DNNSB-induced colitis in rats. Indian J. Pharmacol. 43(6): 656-661.

Lohmann, L. G. (2015). Handroanthus in Flora do Brasil. Jardim Botânico do Rio de Janeiro. 〈http://reflora.jbrj.gov.br/reflora/floradobrasil/FB117466> .

Lucchese, A. M., \& Vale, A. E. (2006). Plantas da Caatinga: Perfil Botânico, Fitoquímica e Atividade Biológica. Associação Plantas do Nordeste, 4.

Maria-Ferreira, D, Carlotto, J, Dallazen, J. L., da Luz, B. B., de Souza, L. M., de Paula Werner, M. F., \& Cipriani, T. R. (2020). A polysaccharide fraction from Handroanthus albus (yellow ipê) leaves with antinociceptive and anti-inflammatory activities. International Journal of Biological Macromolecules, 159, $1004-1012$

Morais, M. G., de Oliveira Junior, A. S., Aguiar, E. L. C. C., Ferreira, M. V. G., Martins, M. F., de Fátima Oliveira, P. P., \& dos Santos Lima, L. A. R. (2020). Triagem fitoquímica e avaliação da atividade antibacteriana das flores de Handroanthus impetiginosus. Biodiversidade, 19.

Mohan, S, Thiagarajan, K, Sundaramoorthy, B, Gurung, V, Barpande, M, Shilpi, A, \& Chandrasekaran, R (2016). Alleviation og 4-nitroquinoline 1-oxide induced oxidative strees by Oroxylum indicum (L.) leaf extract in albino Wistar rats. BMC Complemente Altern Med. 16:229.

Moharram, F. A., \& Marzouk, M. S. A. (2007). A novel phenylethanoid dimer and flavonoids from Jacaranda mimosifolia, Z. Naturforsch B. 62, 1213-1220. Parrilha, GL (2012). Complexos metálicos de hidrazonas, tiossemicarbazonas e lapachol: atividade farmacológica e avaliação de relações estrutura-atividade.

Reis, A. C. C., Silva, B. M., Moura, H. M. M., Pereira, G. R., \& Brandão, G. C. (2020). Anti-Zika virus activity and Chemical characterization by ultra-high performance liquid chromatography (UPLC-DAD-UV-MS) of etanol extracts in Tecoma species. BMC Complement Med Ther, 20 , 246. https://doi.org/10.1186/s12906-020-03040-0.

Rocha, K. B. F., Oliveira, C. N., Azevedo, I. M., Macedo, R., \& Medeiros, A. C. (2019). Effect of Arrabidaea chica extract against chemically induced breast cancer in animal model. Act Cir Bras, 30(10): 201901001.

Rocha, C. Q., Vilela, F. C., Cavalcante, G. P., Santa-Cecilia, F. V., Santos-e-Silva, L., dos Santos, M. H., \& Giusti-Paiva, A. (2011). Anti-inflammatory and antinociceptive effects of Arrabidaea brachypoda (DC.) Bureau roots. J. Ethnopharmacol, 133:396-401. 10.1016/j.jep.2010.10.009.

Rocha, K. B. F., Oliveira, C. N., Azevedo, I. M., Macedo, R., \& Medeiros, A. C. (2019). Effect of Arrabidaea chica extract against chemically induced breast cancer in animal model. Act Cir Bras, 30(10): 201901001.

Serra, M. B., Rocha, W. A., Rocha, C., Furtado, P. G. R., Borges, A. C. R., Silva, S. N., Tangerina, M. M. P., Nascimento, J. R., Vilegas, W., Alves, A. C., Barbeiro, DF, Souza, HP, Abreu, IC, Borges, MOR (2020). Chemical characterization and wound healing property of Jacaranda decurrens Cham. (Bignoniaceae): An experimental study based on molecular mechanisms. Evid Based Complement Alternat. Med, 4749712.

Sichaem, J., Kaennakam, S., Siripong, P, \& Tip-pyang, S. (2012). Tabebuialdehydes A-C, cyclopentene dialdehyde derivatives from the roots of Tabebuia rosea. Fitoterapia, 83, pp. 1456-1459.

Zhang, L., Hasegawa, I., \& Ohta, T. (2016). Anti-inflammatory cyclopentene derivatives from the inner bark of Tabebuia avellanedae. Fitoterapia, 109, pp. $217-223$ 